\title{
The effect of weight loss on lameness in obese dogs with osteoarthritis
}

\author{
William G. Marshall • Herman A. W. Hazewinkel • \\ Dermot Mullen • Geert De Meyer • Katrien Baert • \\ Stuart Carmichael
}

Accepted: 25 February 2010 /Published online: 17 March 2010

(C) The Author(s) 2010. This article is published with open access at Springerlink.com

\begin{abstract}
This paper describes the effect of weight loss on lameness in obese dogs with osteoarthritis (OA). Fourteen obese client-owned dogs with clinical and radiographic signs of OA participated in an open prospective clinical trial. After a screening visit and a visit for collection of baseline data, the dogs were fed a restricted-calorie diet over a study period of 16 weeks that incorporated six follow-up visits. At each visit, body weight and pelvic circumference were measured and severity of lameness was assessed using a numeric rating scale (NRS), a visual analogue scale (VAS) and kinetic gait analysis. This is the first study to assess both subjectively and objectively, the effect of weight loss alone on lameness in obese dogs with OA. The results indicate that body weight reduction causes a significant decrease in lameness from a weight loss of $6.10 \%$ onwards. Kinetic gait analysis supported the results from a body weight reduction of $8.85 \%$ onwards. These results confirm that weight loss should be presented as an important treatment modality to owners of obese dogs with OA and that noticeable improvement may be seen after modest weight loss in the region of $6.10-8.85 \%$ body weight.
\end{abstract}

Keywords Dog $\cdot$ Osteoarthritis $\cdot$ Obesity $\cdot$ Weight loss $\cdot$ Lameness

\footnotetext{
W. G. Marshall $(\bowtie) \cdot$ S. Carmichael

Small Animal Hospital, University of Glasgow Veterinary School, Bearsden Road, Glasgow G61 1QH Scotland, UK

e-mail: w.marshall@vet.gla.ac.uk

H. A. W. Hazewinkel

Department of Clinical Sciences of Companion Animals, Faculty of Veterinary Medicine, Utrecht University, Yalelaan 108, 3508 TD Utrecht, The Netherlands

D. Mullen

The Peoples Dispensary for Small Animals, 1a-1b Shamrock Street, G4 9JZ Glasgow, Scotland, UK

G. De Meyer $\cdot$ K. Baert

Stat-Gent CRESCENDO, Ghent University, Krijgslaan 281 S9, 9000 Ghent, Belgium
} 


\section{Introduction}

Obesity and osteoarthritis (OA) are recognized as two significant health problems that affect domestic dogs worldwide. It has been estimated that $20 \%$ of adult dogs have OA (Johnston 1997) and previous studies have given figures for canine obesity prevalence of 24-41\% (Edney and Smith 1986; McGreevy et al. 2005). In humans, obesity has been identified as a causative factor in the development of knee and hand OA (Grotle et al. 2008; Reijman et al. 2007; Dahaghin et al. 2007). The work of Kealy and colleagues (2000) suggests that obesity may be a risk factor for the development of OA in the dog and this relationship is most convincing for hip OA secondary to dysplasia (Smith et al. 2006). The relationship between obesity and $\mathrm{OA}$ in humans and in dogs potentially involves mechanical, metabolic and biochemical factors and has been reviewed (Marshall et al. 2009; Radin et al. 2009). According to a recent meta-analysis, obese people with knee OA enjoy significantly improved mobility following a body weight reduction of just $5.1 \%$ (Christensen et al. 2007). Three previous studies have demonstrated both subjectively and objectively, that weight loss in obese dogs with OA (of various joints) results in improved mobility (Burkholder and Hulse 2000; Impellizeri et al. 2000; Mlacnik et al. 2006), though all of these studies have limitations. The first used kinetic gait analysis alone (Burkholder and Hulse 2000). The second used only subjective outcome measures (Impellizeri et al. 2000). The third demonstrated improvement using subjective outcome measures and kinetic gait analysis, but evaluated weight loss in combination with physiotherapy and it is difficult to determine their relative effects (Mlacnik et al. 2006). The aim of the study presented here was to investigate both subjectively and objectively the effect of weight loss alone on lameness in obese dogs with hip and / or elbow OA. On the basis of the three previous studies (Burkholder and Hulse 2000; Impellizeri et al. 2000; Mlacnik et al. 2006), the hypothesis was that an improvement in lameness would be observed, and we aimed to determine the minimum amount of weight loss that would provide a significant improvement.

\section{Materials and methods}

\section{Study population}

Client-owned adult dogs of any breed and both sexes, entire or neutered, and with clinical signs of lameness in one or more limbs were considered for inclusion in the study. Dogs that had radiographic evidence of hip and / or elbow OA and had a body weight at least $20 \%$ above their ideal body weight (obese dogs) were eligible for inclusion. Ideal body weight for each individual dog was estimated using breed standards for body size and weight (Burkholder and Toll 2000). Exclusion criteria included abnormal findings on haematology or serum biochemistry, other orthopaedic or systemic abnormalities and treatment with non-steroidal anti-inflammatory drugs three weeks prior to or during the study. Owners of all dogs included in the study signed an informed consent form.

Study design

The study consisted of a weight loss program with regular assessment of the effect of weight loss on lameness. There were eight visits in total over a period of 18 weeks; the first six spaced two weeks apart and the last two spaced four weeks apart. 
At the screening visit, potentially suitable cases were evaluated to see if they met all the inclusion criteria and none of the exclusion criteria. Body weight and pelvic circumference were measured. Pelvic circumference was measured at a point cranial to the ilium, at the level of the sixth lumbar vertebra. This was followed by a general clinical examination, routine haematology and biochemistry and complete orthopaedic examination. Subjective and objective lameness assessments were performed. Radiographs were taken (under sedation) of hip and / or elbow joints identified as potentially affected by OA.

For the subjective lameness assessment, numerical rating scale (NRS) and visual analogue scale (VAS) lameness scores were given for the walk and trot. Where multiple limb lameness was present, the worst affected limb was selected by the investigator at the screening visit and a lameness score was given for this limb only. The following six grades of lameness severity for NRS were used: $0=$ clinically sound; $1=$ barely detectable lameness; 2=mild lameness; 3=moderate lameness; 4=severe lameness (carries limb intermittently when trotting); and $5=$ could not be more lame (non-weight bearing). A dog was classified as "improved" if it showed a decrease of at least one grade in at least one of the two NRS lameness scores (walking or trotting). For the VAS lameness score a $100 \mathrm{~mm}$ long horizontal bar was labeled with "clinically sound" (0) at one end and "could not be more lame" (100) at the other end. The investigator made a mark on the VAS that best represented the lameness of the dog.

For the objective lameness assessment a force-plate (Kistler 9281CA, Kistler Instrumente $\mathrm{AG}$, Winterthur, Switzerland) mounted in a wooden platform measuring $5 \mathrm{~m} \times 0.9 \mathrm{~m} \times 0.2 \mathrm{~m}$ and accompanying data acquisition system (DAQ System Type 5691, Kistler Instrumente AG, Winterthur, Switzerland) and software (Bioware Version 4.0, Kistler Instrumente AG, Winterthur, Switzerland) were used to measure ground reaction forces. It was attempted to trot each dog over the force-plate in such way that only one forefoot followed by the ipsilateral hindfoot contacted the plate at each trial. Velocity was measured using two photoelectric cells placed $2 \mathrm{~m}$ apart connected to a stopwatch (custom design, University of Glasgow, UK). For each dog, velocity was maintained within a $+/-0.3 \mathrm{~m} / \mathrm{s}$ range for every trial recorded. From the force-time graph generated for each trial, peak vertical force (PFz), peak braking force (PFy) and vertical impulse (IFz) were obtained and recorded for fore and hindlimb. Force was expressed as Newtons(N)/kg body weight.

Two weeks after the screening visit the weight loss program started (baseline visit, designated visit 1). Based on estimated ideal body weight, caloric intake was calculated to achieve between 0.5 and $1 \%$ body weight loss per week. Prescription diet foods formulated to achieve weight loss (Royal Canin Obesity Management ${ }^{\circledR}$ or Hills' $\mathrm{R} / \mathrm{D}^{\circledR}$ ) were used in all cases in accordance with the instructions of the manufacturer. For each dog included in the study, the owner was instructed not to change the patients' level of exercise for the duration of the weight loss program. At visit 1 and each subsequent visit, body weight, pelvic circumference and lameness were reassessed. All clinical examinations and lameness assessments were made by the same person (WGM).

\section{Data analysis}

Evolution of outcome measures over time was analyzed using linear mixed models (Fitzmaurice et al. 2004) with structures based on model validity checks. For changes in body weight and pelvic circumference versus visit 1, a model with visit-specific averages was combined with a first order autoregressive structure over time for the errors. For VAS walking and trotting lameness scores, averages were modeled as a linear function of time 
with random intercepts for each dog, combined with a first order autoregressive structure over time for the errors. PFz, PFy and IFz were modeled as a linear function of time with random intercepts for each dog allowing different trajectories for the worst affected limb, its contralateral limb and for the two other limbs. Correlation between repeated measurements was acknowledged using a compound symmetry structure. For all outcome measures a likelihood ratio test was performed to verify homogeneity depending on whether the worst affected limb was a forelimb or a hindlimb.

A non-parametric exact paired Wilcoxon test was used to compare NRS walking and trotting lameness scores measured at visit 1 to those obtained at each subsequent visit. Homogeneity between dogs with the worst affected limb being a forelimb or a hindlimb was verified at baseline (visit 1) using an exact Wilcoxon test.

Unless stated otherwise, the significance level of confidence intervals and performed tests is 5\%. All statistical analyses were performed using a statistical software package (SAS, version 9.2, SAS Institute, Inc.)

\section{Results}

A total of sixteen dogs were enrolled in the study. Two dogs were excluded from the analysis because they were lost to follow up after the screening visit. Fourteen dogs were included in the analysis. The dogs weighed between 20.7 and $60.9 \mathrm{~kg}$ and were 20 to $34 \%$ above their ideal body weight with an average of $23.8 \%$. There were six Labradors, two mixed breed dogs, two Staffordshire Bull Terriers, one Rottweiler, Border Collie, Bearded Collie and Springer Spaniel recruited. The age of the dogs ranged from 10 months to 13 years with a mean of 6.8 years. Five dogs had bilateral hip dysplasia and secondary OA. Two dogs had unilateral hip dysplasia and secondary OA and one of these had a total hip replacement on the contralateral side. Five dogs had bilateral elbow OA. An inciting cause could be definitively identified in only one dog with a chronic ununited anconeal process (UAP). One dog had bilateral hip and bilateral elbow OA, and one bilateral elbow and unilateral hip OA. Both of these dogs with combined hip and elbow OA displayed forelimb lameness primarily. The worst affected limb was a forelimb in seven dogs and a hindlimb in the other seven dogs.

Body weight (loss) and pelvic circumference (reduction) at each visit are summarized in Table 1. At the end of the study, dogs had loss on average $8.60 \%$ of their initial body weight and $6.92 \%$ of their initial pelvic circumference.

Starting from visit 3 (Tables 2 and 3, Fig. 1), body weight at the successive visits was significantly different from the first visit $(\mathrm{p} \leq 0.0004)$. At the end of the study, dogs had lost on average $8.85 \%$ of their initial body weight (model-based estimate, $95 \%$ confidence interval (CI) $[6.82 \%, 10.88 \%])$, which is significantly different from the initial body weight $(\mathrm{p}<0.0001)$. An approximate average reduction in body weight of $0.55 \%(95 \%$ CI $[0.43 \%$, $0.68 \%]$ ) per week was attained. Starting from visit 5 , pelvic circumference was significantly different from the first visit $(\mathrm{p}<0.0001)$ (Tables 2 and 3, Fig. 1). At visit 7, the pelvic circumference had on average reduced by $6.76 \%$ (model-based estimate, $95 \%$ CI $[4.07 \%$, $9.45 \%]$ ). For body weight loss and pelvic circumference reduction, there was no difference depending on whether the worst affected limb was a fore or a hindlimb $(\mathrm{p}=0.9103$ and $\mathrm{p}=$ 0.3594 , respectively).

Average VAS lameness scores for both walking and trotting were significantly different from the first visit starting from visit 2 onwards $(\mathrm{p}<0.0001$ and $\mathrm{p}=0.0005$, respectively; Tables 2 and 3, Fig. 2). An average decrease in VAS lameness score per week was 
Table 1 Descriptive statistics for outcome measures body weight (loss), pelvic circumference (reduction) and lameness scores (NRS and VAS) over time

\begin{tabular}{|c|c|c|c|c|c|c|c|c|}
\hline \multirow{2}{*}{\multicolumn{2}{|c|}{ Variable }} & \multicolumn{7}{|l|}{ Visit } \\
\hline & & 1 (day 0 ) & 2 (day 14) & 3 (day 28) & 4 (day 42) & 5 (day 56) & 6 (day 84) & 7 (day 112) \\
\hline \multicolumn{2}{|c|}{ Body weight $(\mathrm{kg})^{\mathrm{A}}$} & $34.4 \pm 9.8$ & $34.7 \pm 9.4$ & $35.0 \pm 9.6$ & $30.8 \pm 7.5$ & $33.3 \pm 9.6$ & $33.1 \pm 10.0$ & $32.8 \pm 9.6$ \\
\hline \multicolumn{2}{|c|}{ Body weight loss (\%) ${ }^{\mathrm{A}}$} & / & $1.66 \pm 2.63$ & $3.13 \pm 2.33$ & $4.56 \pm 3.47$ & $6.27 \pm 3.40$ & $8.14 \pm 3.34$ & $8.60 \pm 4.97$ \\
\hline \multicolumn{2}{|c|}{$\begin{array}{l}\text { Pelvic circumference } \\
(\mathrm{cm})^{\mathrm{A}}\end{array}$} & $71.9 \pm 8.2$ & $70.8 \pm 7.2$ & $71.2 \pm 6.5$ & $67.2 \pm 3.9$ & $68.6 \pm 6.6$ & $67.5 \pm 8.0$ & $68.0 \pm 7.6$ \\
\hline \multicolumn{2}{|c|}{$\begin{array}{l}\text { Pelvic circumference } \\
\text { reduction }(\%)^{\mathrm{A}}\end{array}$} & / & $2.26 \pm 4.70$ & $2.72 \pm 5.01$ & $2.57 \pm 5.92$ & $6.15 \pm 4.12$ & $7.83 \pm 3.61$ & $6.92 \pm 5.58$ \\
\hline \multirow{3}{*}{$\begin{array}{l}\text { NRS lameness } \\
\text { score }\end{array}$} & Walking $^{\mathrm{B}}$ & $2(1-3)$ & $2(1-3)$ & $2(1-3)$ & $2(0-4)$ & $2(0-3)$ & $1(0-3)$ & $1(0-3)$ \\
\hline & Trotting ${ }^{\mathrm{B}}$ & $2(1-3)$ & $2(0-3)$ & $2(0-3)$ & $2(0-4)$ & $2(0-3)$ & $1(0-3)$ & $1(0-3)$ \\
\hline & Combined $^{\mathrm{C}}$ & / & $31 \%$ & $45 \%$ & $56 \%$ & $67 \%$ & $73 \%$ & $82 \%$ \\
\hline \multirow{2}{*}{$\begin{array}{l}\text { VAS lameness } \\
\text { score }^{\mathrm{A}}\end{array}$} & Walking & $38.5 \pm 11.7$ & $37.5 \pm 14.5$ & $35.3 \pm 9.8$ & $33.6 \pm 20.5$ & $25.8 \pm 14.1$ & $23.6 \pm 11.0$ & $22.2 \pm 9.9$ \\
\hline & Trotting & $36.5 \pm 14.4$ & $32.5 \pm 18.2$ & $32.2 \pm 14.2$ & $31.8 \pm 23.8$ & $25.0 \pm 14.0$ & $24.9 \pm 15.5$ & $23.2 \pm 14.2$ \\
\hline \multicolumn{9}{|c|}{ A : Summary given as mean \pm standard deviation } \\
\hline \multicolumn{9}{|c|}{ B : Summary given as median (range) } \\
\hline
\end{tabular}

estimated to be 1.14 for walking (model-based estimate, 95\% CI $[0.66,1.63]$ ) and 0.88 for trotting (model-based estimate, 95\% CI [0.40, 1.36]), which is significantly different from 0 . For walking and trotting VAS lameness scores, there was no difference depending on whether the worst affected limb was a fore or a hindlimb $(p=0.1003$ and $p=0.3499$, respectively).

At the end of the study, $82 \%$ of the dogs showed an improvement in the combined NRS lameness score (Table 1). At visit 5, a significant difference in NRS lameness score compared to visit 1 was detected for trotting $(\mathrm{p}=0.0313$, Table 3$)$. By the end of the study there was also a significant difference compared to visit 1 for the NRS lameness score for walking ( $p=0.0273$, Table 3 ). At baseline (visit 1 ), there was no difference depending on whether the worst affected limb was a fore or a hindlimb $(p=0.1841$ and $p=1.0000$ for walking and trotting NRS lameness score, respectively).

The mean subject velocity measured during kinetic gait analysis ranged from 1.36$1.69 \mathrm{~m} / \mathrm{s}$ between visits. For all force-plate outcomes there were significant differences $(\mathrm{p}<$ 0.0001) in PFz, PFy and IFz depending on whether the worst affected limb was a fore or a hindlimb. For dogs whose worst affected limb was a forelimb, baseline (visit 1) PFz, PFy and IFz differ between the worst affected limb and the contralateral $\operatorname{limb}(\mathrm{p}<0.0001, \mathrm{p}=$ 0.0002 and $\mathrm{p}<0.0001$, respectively, Figs. 3, 4 and 5). For these limbs, evolution over time is different or shows a tendency to be different between the worst affected and the contralateral $\operatorname{limb}(p=0.0002, p=0.0726$ and $p=0.0030$ for PFz, PFy and IFz, respectively). The worst affected limb improves significantly over time for PFz and IFz $(p<0.0001$ and $\mathrm{p}=0.0047)$ but not for PFy $(\mathrm{p}=0.1698)$. The PFy of the hindlimbs decreased significantly over time $(\mathrm{p}=0.0216)$ while there is no evidence for changes in the hindlimbs for PFz and $\mathrm{IFz}(\mathrm{p}=0.5425$ and $\mathrm{p}=0.4007$, respectively). These evolutions show that the estimated difference between the worst affected forelimb and the contralateral forelimb reduces by at least $50 \%$ for $\mathrm{PFz}, \mathrm{PFy}$ and $\mathrm{IFz}$ at visit 6 , and by at least $80 \%$ for PFz and IFz at visit 7 (Table 4). 


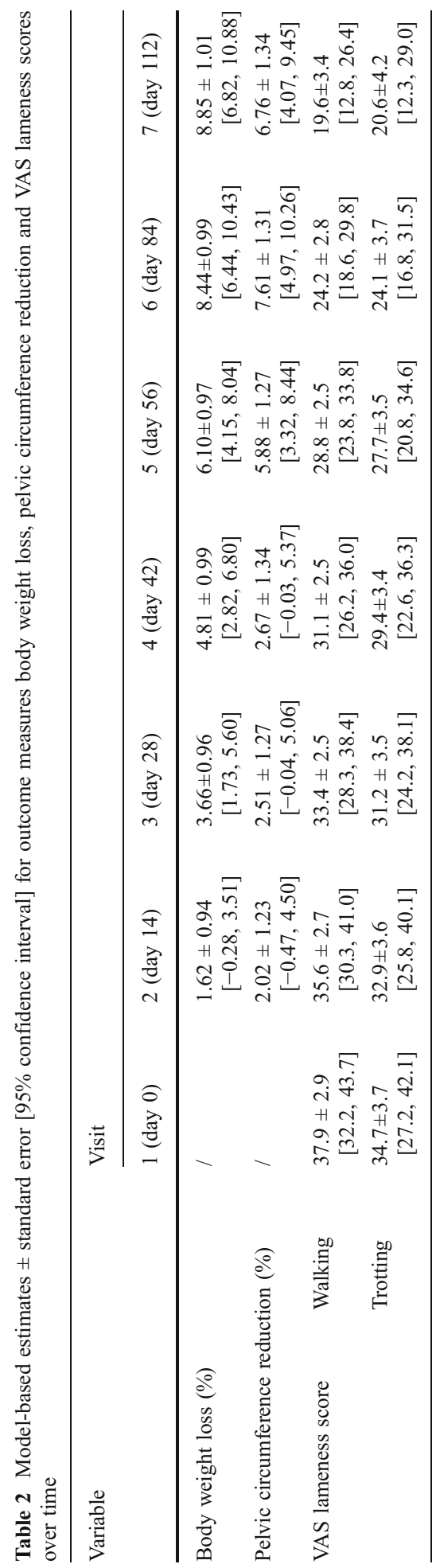


Table 3 Statistical hypothesis test results for the outcome measures body weight (loss), pelvic circumference (reduction) and lameness scores (NRS and VAS) at each visit compared to visit 1

\begin{tabular}{|c|c|c|c|c|c|c|c|}
\hline \multirow{2}{*}{\multicolumn{2}{|c|}{ Variable }} & \multicolumn{6}{|l|}{ Visit } \\
\hline & & 2 (day 14$)$ & 3 (day 28 ) & 4 (day 42) & 5 (day 56) & 6 (day 84) & 7 (day 112 ) \\
\hline \multicolumn{2}{|c|}{ Body weight loss (\%) ${ }^{\mathrm{A}}$} & $0.0922 \mathrm{~ns}$ & $0.0004 * * *$ & $<0.0001 * * *$ & $<0.0001 * * *$ & $<0.0001 * * *$ & $<0.0001 * * *$ \\
\hline \multicolumn{2}{|c|}{$\begin{array}{l}\text { Pelvic circumference } \\
\text { reduction }(\%)^{\mathrm{A}}\end{array}$} & $0.1091 \mathrm{~ns}$ & $0.0533 \mathrm{~ns}$ & $0.0525 \mathrm{~ns}$ & $<0.0001 * * *$ & $<0.0001 * * *$ & $<0.0001 * * *$ \\
\hline \multirow{2}{*}{$\begin{array}{l}\text { NRS } \\
\text { lameness } \\
\text { score }\end{array}$} & Walking $^{\mathrm{B}}$ & $1.0000 \mathrm{~ns}$ & $0.6250 \mathrm{~ns}$ & $0.5625 \mathrm{~ns}$ & $0.1094 \mathrm{~ns}$ & $0.0781 \mathrm{~ns}$ & $0.0273 *$ \\
\hline & Trotting $^{\mathrm{B}}$ & $0.2500 \mathrm{~ns}$ & $0.1250 \mathrm{~ns}$ & $0.7500 \mathrm{~ns}$ & $0.0313^{*}$ & $0.0352 *$ & $0.0195 *$ \\
\hline \multirow{2}{*}{$\begin{array}{l}\text { VAS } \\
\text { lameness } \\
\text { score }^{\mathrm{A}}\end{array}$} & Walking & $<0.0001 * * *$ & $<0.0001 * * *$ & $<0.0001 * * *$ & $<0.0001 * * *$ & $<0.0001 * * *$ & $<0.0001 * * *$ \\
\hline & Trotting & $0.0005^{* * *}$ & $0.0005^{* * *}$ & $0.0005^{* * *}$ & $0.0005^{* * *}$ & $0.0005^{* * *}$ & $0.0005^{* * *}$ \\
\hline
\end{tabular}

A : Statistical test in a linear mixed model

B : Non-parametric exact paired Wilcoxon test

*: Statistically significant at $\alpha=5 \%$ versus visit 1

***: Statistically significant at $\alpha=0.1 \%$ versus visit 1

ns: Not significant

For dogs whose worst affected limb was a hindlimb, there is no difference in ground reaction forces between the worst affected limb and the contralateral limb at baseline (visit 1 , $\mathrm{p}=0.3852, \mathrm{p}=0.8130$ and $\mathrm{p}=0.1429$ for PFz, PFy and IFz, respectively).

\section{Discussion}

In the dog, obesity and overweight are arbitrarily defined as a body weight $20 \%$ and $10 \%$ greater than ideal, respectively (Burkholder and Toll 2000). Previous studies examining

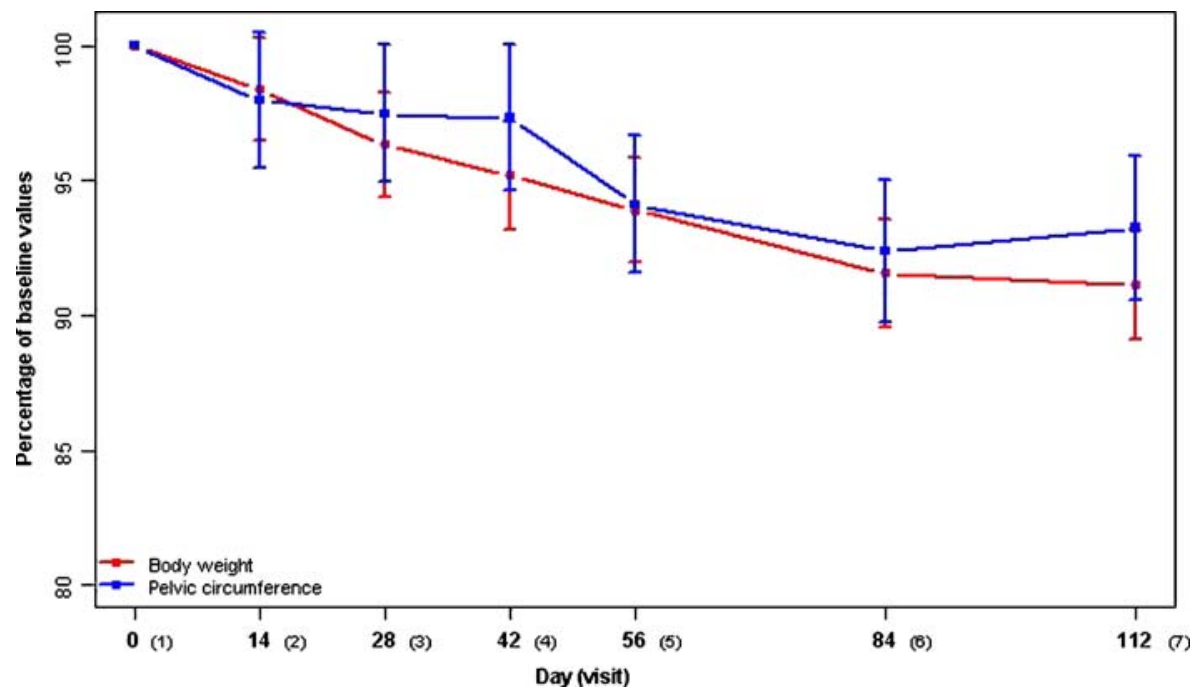

Fig. 1 Average percentage of baseline values for body weight and pelvic circumference at each visit, with model-based estimates and their $95 \%$ confidence intervals 


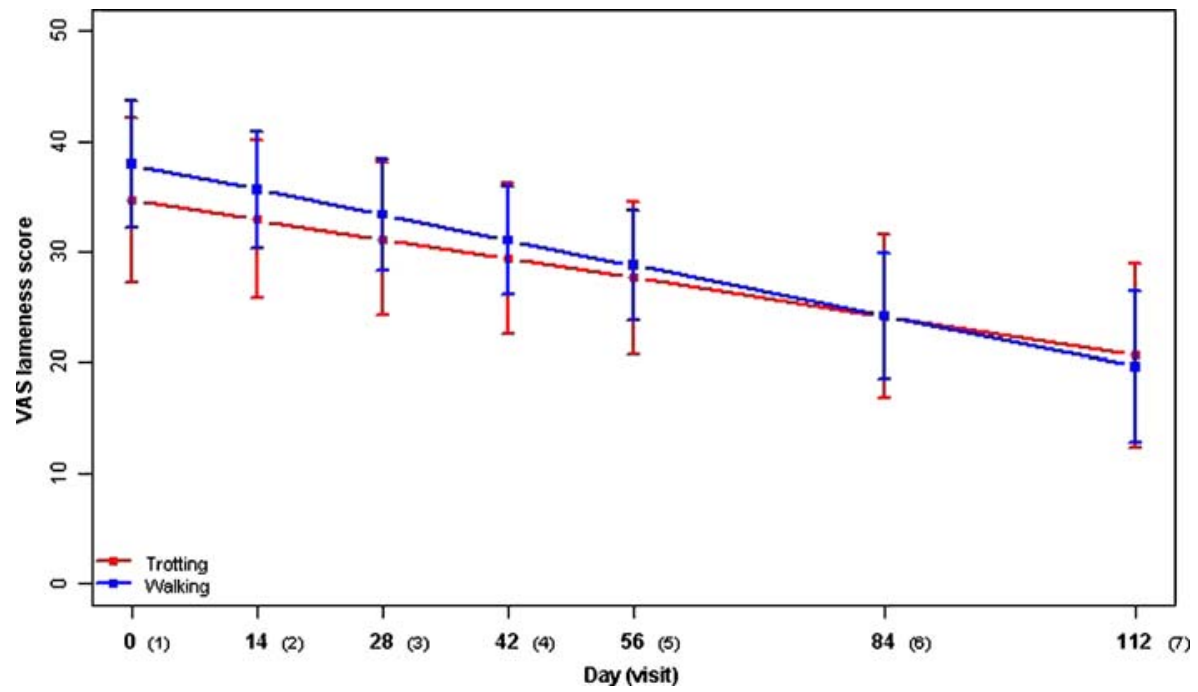

Fig. 2 Evolution of average VAS lameness scores with model-based estimates and their 95\% confidence intervals

weight loss in osteoarthritic dogs have included subjects that ranged from 11-29\% overweight (Burkholder and Hulse 2000; Impellizeri et al. 2000). The current study included only obese dogs, estimated to be $20 \%$ greater than ideal body weight. It was expected that weight loss would have its most significant effect on lameness in obese dogs with OA. Furthermore, in humans, it is the obese (body mass index greater than 30) population with OA that tends to be the focus of research examining the beneficial effect of weight loss (Christensen et al. 2007; Hooper et al. 2007; Christensen et al. 2005). A

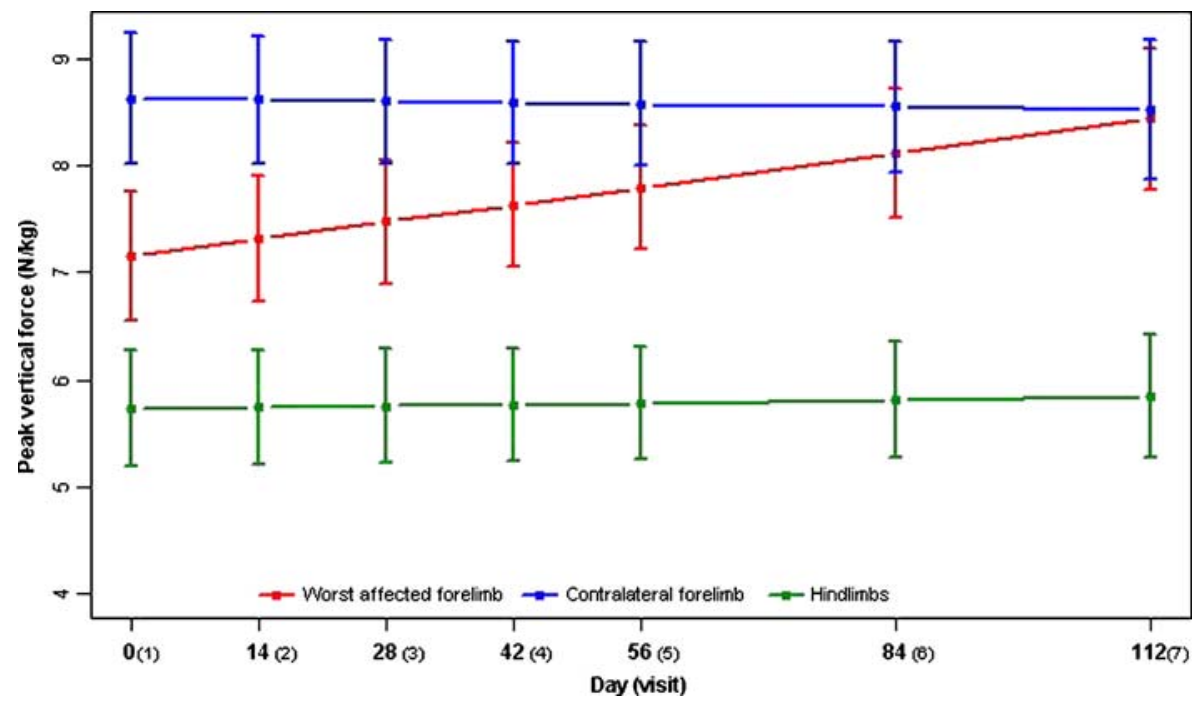

Fig. 3 Evolution of average peak vertical force (model-based estimates and their 95\% confidence intervals) for dogs with elbow OA and forelimb lameness broken down by limb type 


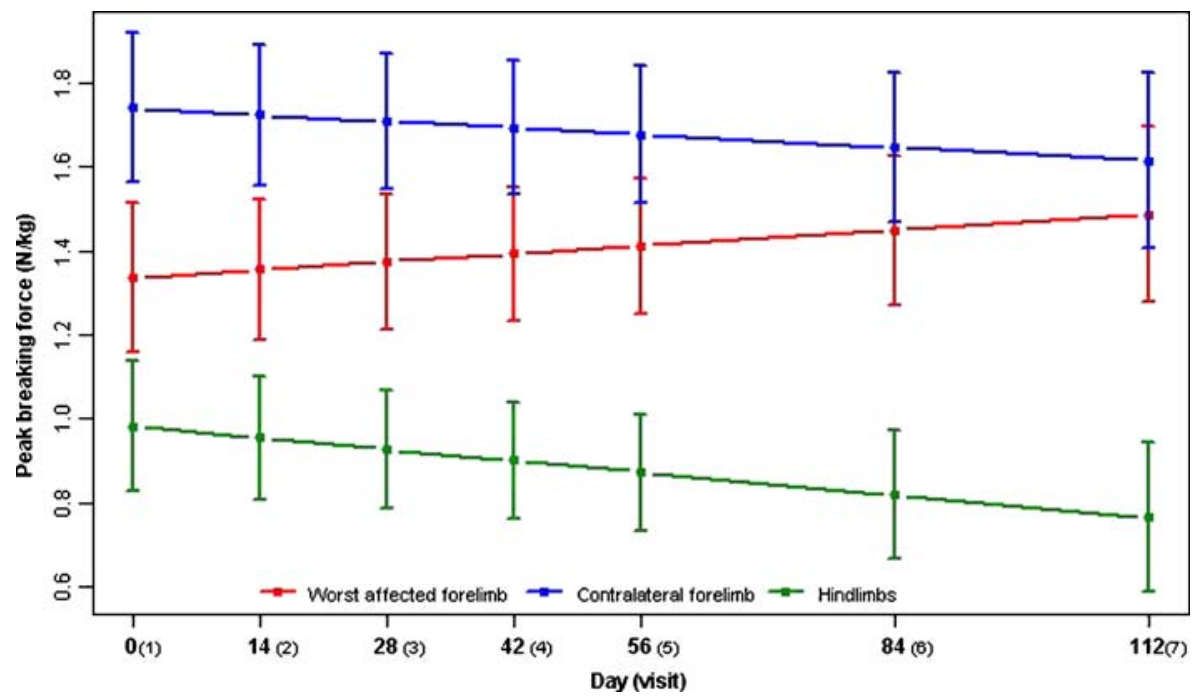

Fig. 4 Evolution of average peak breaking force (model-based estimates and their 95\% confidence intervals) for dogs with elbow OA and forelimb lameness broken down by limb type

limitation of the study reported here is the small number of obese dogs recruited - only fourteen completed sufficient visits for data analysis. Stimulating and maintaining client interest in canine weight loss programs can be challenging (Burkholder and Toll 2000) and this hindered recruitment of cases. Inclusion of multiple centres in any future studies of a similar nature should be considered to maximize sample size.

In two of the previous weight loss studies the dogs enrolled were limited to those with hip dysplasia and secondary OA (Burkholder and Hulse 2000; Impellizeri et al. 2000). By

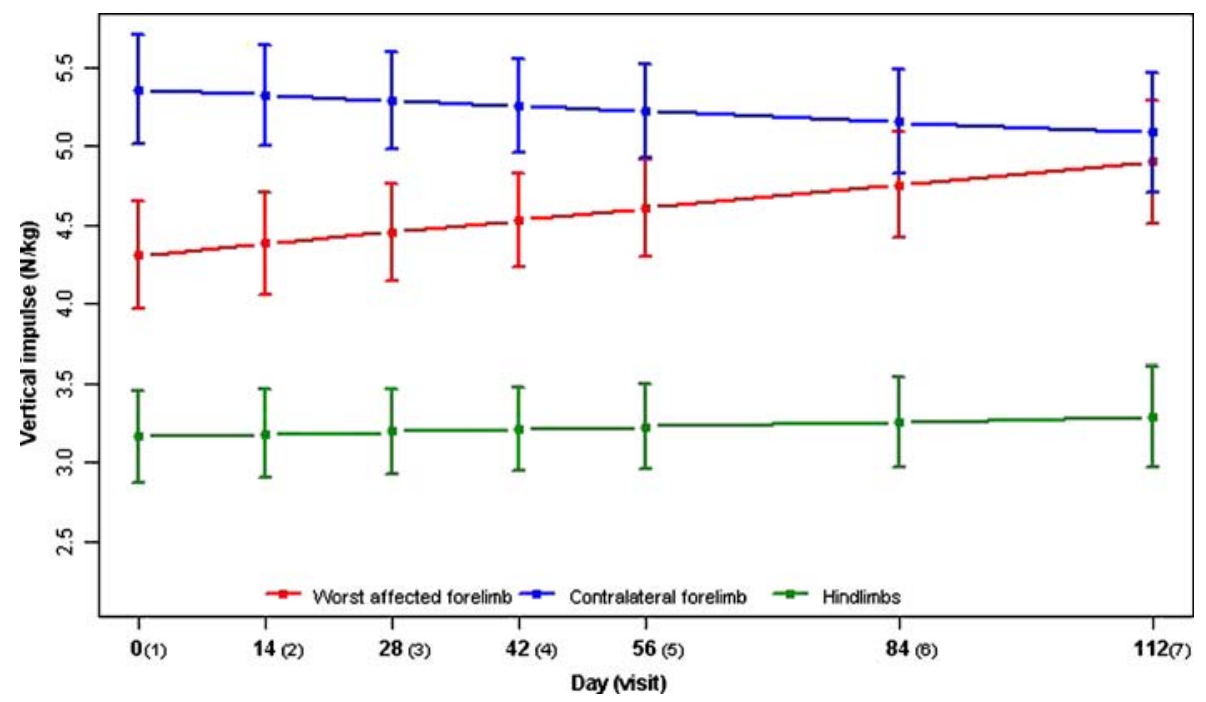

Fig. 5 Evolution of average vertical impulse (model-based estimates and their $95 \%$ confidence intervals) for dogs with elbow OA and forelimb lameness broken down by limb type 
Table 4 Model-based estimates \pm standard error for the difference between the worst affected and contralateral limb for peak vertical force (PFz), peak braking force (PFy) and vertical impulse (IFz) over time for dogs whose worst affected limb is a forelimb

\begin{tabular}{|c|c|c|c|c|c|c|c|c|}
\hline \multirow[t]{2}{*}{ Variable } & & \multicolumn{7}{|l|}{ Visit } \\
\hline & & 1 (day 0) & 2 (day 14 ) & 3 (day 28 ) & 4 (day 42) & 5 (day 56) & 6 (day 84) & 7 (day 112) \\
\hline \multirow{3}{*}{$\begin{array}{l}\text { Ground reaction force } \\
(\mathrm{N} / \mathrm{kg})\end{array}$} & $\mathrm{PFz}$ & $1.48 \pm 0.29$ & $1.30 \pm 0.27$ & $1.13 \pm 0.25$ & $0.96 \pm 0.25$ & $0.78 \pm 0.25$ & $0.44 \pm 0.28 *$ & $0.09 \pm 0.34 * *$ \\
\hline & PFy & $0.40 \pm 0.11$ & $0.37 \pm 0.10$ & $0.34 \pm 0.09$ & $0.30 \pm 0.09$ & $2.67 \pm 0.09$ & $0.20 \pm 0.11^{*}$ & $0.13 \pm 0.13^{*}$ \\
\hline & $\mathrm{IFz}$ & $1.05 \pm 0.18$ & $0.94 \pm 0.16$ & $0.83 \pm 0.14$ & $0.72 \pm 0.14$ & $0.62 \pm 0.14$ & $0.40 \pm 0.17^{*}$ & $0.18 \pm 0.22 * *$ \\
\hline
\end{tabular}

*: The difference estimate is reduced by at least $50 \%$ as compared to the difference estimate at visit 1

**: The difference estimate is reduced by at least $80 \%$ as compared to the difference estimate at visit 1

contrast, the most recent study (Mlacnik et al. 2006) included dogs with OA of the hip, elbow, shoulder and stifle. The dogs of the study reported here had between one and four affected hip and / or elbow joints. In all cases but one (with an obvious UAP) the (suspected) underlying developmental abnormality causing elbow OA could not be definitively diagnosed. The fact that a positive response to weight loss was seen in this somewhat heterogeneous experimental population suggests that our results are relevant to the wider population of dogs with hip and / or elbow OA (Thrusfield 1995).

It has been stated that to maintain owner's interest and compliance to complete a canine weight loss program within a reasonable time frame a minimum of $0.5 \%$ body weight should be lost per week (Burkholder and Toll 2000). Conversely, a rate of weight loss greater than $2 \%$ per week is considered unhealthy, since this may result in loss of lean tissue (Burkholder and Toll 2000). In this study a target of $0.5-1 \%$ per week was set and achieved, with $0.55 \%$ body weight lost on average. At the end of the 16 -week weight loss period the average body weight reduction was $8.85 \%$. This is comparable to $9.3 \%$ body weight loss reported by Mlacnik and co-workers (2006) for dogs with OA at the conclusion of a six month caloric restriction program combined with moderate physiotherapy. In that study, a separate group of dogs underwent intense physiotherapy and lost $13.6 \%$ of their body weight over six months which was attributed to increased owner compliance and physical activity. In the studies by Impellizeri et al. (2000) and Burkholder and Hulse (2000) body weight reductions of $11-18 \%$ and $3.9-12 \mathrm{~kg}$ were achieved. In the former study the weight loss period was 10-19 weeks; in the latter the time period is not given and all dogs reached their ideal body weight. Pelvic circumference is an objective measure that changes significantly with body weight and is proportional to the amount of body fat in both cats and dogs (Burkholder and Toll 2000). In the study reported here a statistically significant decrease in pelvic circumference was seen with decreasing body weight.

A significant decrease in NRS lameness score at trot and walk were seen beginning at visits 5 and 7 , following a mean body weight decrease of 6.10 and $8.85 \%$, respectively. It has been shown, using an ovine model, that the NRS method of lameness assessment is relatively insensitive (Welsh et al. 1993), and therefore to increase sensitivity, we combined the NRS lameness scores and defined "improvement" as a decrease in lameness score by one grade for either walk or trot. By this method $56 \%$ of dogs had improved by visit 4 ( $4.81 \%$ body weight reduction), and $82 \%$ had improved by the end of the study. A statistically significant decrease in VAS lameness score was seen starting at visit 2, two weeks after starting the weight loss program, with on average $1.62 \%$ body weight lost. Examination of the NRS and VAS results together, suggests that a statistically significant 
improvement in lameness was seen beginning at visit 5 (6.10\% body weight reduction). This is comparable to the results of Impellizeri et al. (2000) where significant improvement in NRS and VAS lameness scores were seen after a mean body weight decrease of $2.4 \mathrm{~kg}$ $(6.2 \%)$ in overweight and obese dogs with hip OA. In the study that compared moderate and intense physiotherapy in combination with weight loss, a significant decrease in NRS lameness score was seen in both groups of dogs following body weight reduction of approximately 5\% (Mlacnik et al. 2006).

In the dogs with bilateral elbow $\mathrm{OA}$ and forelimb lameness, significant asymmetry between contralateral limbs was demonstrated for PFz, PFy and IFz at the start of the study. In the worst affected limb there was a significant increase in PFz and IFz with weight loss, and an increase in PFy that tended towards significance. By visit 7 (8.85\% body weight reduction) the mean difference in PFz, PFy and IFz between forelimbs was in line with previously reported forelimb ground reaction force values for normal dogs (Rumph et al. 1994). This suggests that after body weight reduction of $8.85 \%$ the ground reaction forces measured in the worst affected limb had increased such that the degree of symmetry between forelimbs had returned within normal limits. The significant decrease in hindlimb PFy in dogs with forelimb lameness is interesting. It is recognized that the forelimb is primarily responsible for braking, and the hindlimb for propulsion (Budsberg et al. 1987). It is possible that in dogs with forelimb OA and lameness, a relatively greater proportion of braking is performed by the hindlimbs. The decrease in hindlimb and increase in forelimb PFy seen with weight loss may reflect a shift in the distribution of braking force back to the forelimbs. Because of equipment limitations, it was not possible to measure subject acceleration during kinetic gait analysis in this study. According to previous work, this could only have influenced the results for forelimb PFy as acceleration has no effect on forelimb PFz or IFz, or on hindlimb PFy (Budsberg et al. 1999).

In this study, the improvement in NRS and VAS lameness scores was not reflected in the force-plate results in the dogs with hindlimb lameness - there was no change in $\mathrm{PFz}, \mathrm{IFz}$ or PFy with weight loss. There is a degree of similarity between these results and some of those reported by Mlacnik et al. (2006). In that study, subjective improvement in lameness was first demonstrated among the dogs enrolled in the protocol of weight loss combined with moderate physiotherapy after 60 days and this improvement was maintained throughout the remainder of the study period at days 120 and 180. However, subjective improvement was only confirmed by the force-plate results at day 120 , and then only by the symmetry index for PFz, not for IFz. By contrast, the dogs that were enrolled in the protocol of weight loss and underwent intensive physiotherapy showed a consistent improvement in $\mathrm{PFz}$ and IFz symmetry indices starting from day 60, though it is unclear whether the intensity of physiotherapy or faster rate of weight loss was the important factor underlying this difference. The majority of the dogs in our study with hindlimb lameness had bilateral hip OA, and it was often difficult to determine subjectively which leg was worst affected. It is therefore unsurprising that the ground reaction forces were not significantly different between contralateral limbs. In addition to a lack of asymmetry, there was no change in absolute ground reaction force values produced by either hindlimb following weight loss in our study. This differs from the results of Burkholder and Hulse (2000) who report average increases in PFz of up to $0.98 \mathrm{~N} / \mathrm{kg}$ in dogs with hip OA following weight loss to ideal body weight. Fourier analysis of force-time curves obtained from dogs with hindlimb lameness in this study could prove interesting. This method can reveal subtle differences between curves that are not necessarily apparent by conventional analyses (Katic et al. 2009). The more demonstrable benefit of weight loss in obese dogs with forelimb versus hindlimb lameness in the current study may be related to the fact that 
around $60 \%$ of canine body weight is borne by the forelimbs (Budsberg et al. 1987). Because of this asymmetrical weight distribution, for a given decrease in bodyweight a more significant decrease in pain may be experienced by dogs with forelimb OA.

This is the first study to assess both subjectively and objectively, the effect of weight loss alone on lameness in obese dogs with hip and / or elbow OA. The results indicate that body weight reduction will cause a decrease in clinical signs of lameness, and a significant effect was seen from a weight loss of $6.10 \%$ onwards. Kinetic gait analysis supported the results for dogs with elbow OA and forelimb lameness from a body weight reduction of $8.85 \%$ onwards. These results confirm that weight loss should be presented as an important treatment modality to owners of obese dogs with hip and / or elbow OA and that noticeable improvement may be seen after modest weight loss in the region of $6.10-8.85 \%$ body weight.

Acknowledgements The authors would like to thank Miss Sarah Kelly RVN for her assistance, and the veterinary surgeons that referred cases for inclusion in the study.

Open Access This article is distributed under the terms of the Creative Commons Attribution Noncommercial License which permits any noncommercial use, distribution, and reproduction in any medium, provided the original author(s) and source are credited.

\section{References}

Budsberg SC, Verstraete MC, Soutas-Little RW (1987) Force plate analysis of the walking gait in healthy dogs. American Journal of Veterinary Research 48: 915-918.

Budsberg SC, Rytz U, Johnson SA (1999) Effects of acceleration on ground reaction forces collected in healthy dogs at a trot. Veterinary and Comparative Orthopaedics and Traumatology 12: 15-19.

Burkholder WJ, Hulse DA (2000)Weight loss to optimal body condition increases ground reactive forces in dogs with osteoarthritis (abstract) Proceedings Purina Nutrition Forum; 74.

Burkholder WJ, Toll PW (2000) Obesity. In: Hand MS, Thatcher CD, Remillard RL, Roudebush P (Ed). Small Animal Clinical Nutrition, pp 404-406.

Christensen R, Astrup A, Bliddal H (2005) Weight loss: the treatment of choice for knee osteoarthritis? Osteoarthritis and Cartilage 13: 20-27.

Christensen R, Bartels EM, Astrup A, Bliddal H (2007) Effect of weight reduction in obese patients diagnosed with knee osteoarthritis: a systematic review and meta-analysis. Annals of the Rheumatic Diseases 66: 433-439.

Dahaghin S, Bierma-Zeinstra SMA, Koes BW, Hazes JMW, Pols HAP (2007) Do metabolic factors add to the effect of overweight on hand osteoarthritis? The Rotterdam Study. Annals of the Rheumatic Diseases 66: 916-920.

Edney ATB, Smith PM (1986) Study of obesity in dogs visiting veterinary practices in the United Kingdom. Veterinary Record 118: 391-396.

Fitzmaurice GM, Laird NM, Ware JH (2004) Applied Longitudinal Analysis. In: Wiley Series in Probability and Statistics.

Grotle M, Hagen KB, Natvig B, Dahl FA, Kvien TK (2008) Obesity and osteoarthritis in knee, hip and/or hand: an epidemiological study in the general population with 10 years follow-up. BMC Musculoskeletal Disorders 132: doi:10.1186/1471-2474-9-132.

Hooper MM, Stellato TA, Hallowell PT, Seitz BA, Moskowitz RW (2007) Musculoskeletal findings in obese subjects before and after weight loss following bariatric surgery. International Journal of Obesity 31: 114-120.

Impellizeri JA, Tetrick MA, Muir P (2000) Effect of weight reduction on clinical signs of lameness in dogs with hip osteoarthritis. Journal of the American Veterinary Medical Association 216: 1089-1091.

Johnston SA (1997) Osteoarthritis: joint anatomy, physiology and pathobiology. Veterinary Clinics of North America Small Animal Practice 27: 699.

Katic N, Bockstahler BA, Mueller M, Peham C (2009) Fourier analysis of vertical ground reaction forces in dogs with unilateral hindlimb lameness caused by degenerative disease of the hip joint and in dogs without lameness. American Journal of Veterinary Research 70: 118-126. 
Kealy RD, Lawler DF, Ballam JM, Lust G, Biery DN, Smith GK, Mantz SL (2000) Evaluation of the effect of limited food consumption on radiographic evidence of osteoarthritis in dogs. Journal of the American Veterinary Medical Association 217: 1678-1680.

Marshall WG, Bockstahler B, Hulse D, Carmichael S (2009) Osteoarthritis and obesity - a review: current understanding of the relationship and benefit of obesity treatment and prevention in the dog. Veterinary and Comparative Orthopaedics and Traumatology 22: 339-345.

McGreevy PD, Thomson PC, Pride C, Fawcett A, Grassi T, Jones B (2005) Prevalence of obesity in dogs examined by Australian veterinary practices and the risk factors involved. Veterinary Record 156: 695-702.

Mlacnik E, Bockstahler BA, Muller M, Tetrick MA, Nap RC, Zentek J (2006) Effects of caloric restriction and a moderate or intense physiotherapy program for treatment of lameness in overweight dogs with osteoarthritis. Journal of the American Veterinary Medical Association 229: 1756-1760.

Radin MJ, Sharkey LC, Holycross BJ (2009) Adipokines: a review of biological and analytical principles and an update in dogs, cats, and horses. Veterinary Clinical Pathology 38: 136-156

Reijman M, Pols HAP, Bergink AP, Hazes JMW, Belo JN, Lievense AM, Bierma-Zeinstra SMA (2007) Body mass index associated with onset and progression of osteoarthritis of the knee but not of the hip: The Rotterdam Study. Annals of the Rheumatic Diseases 66: 158-162.

Rumph PF, Lander JE, Kincaid SA, Baird DK, Kammermann JR, Visco DM (1994) Ground reaction force profiles from force platform gait analyses of clinically normal mesomorphic dogs at the trot. American Journal of Veterinary Research 55: 756-761.

Smith GK, Paster ER, Powers MY, Lawler DF, Biery DN, Shofer FS, McKelvie PJ, Kealy RD (2006) Lifelong diet restriction and radiographic evidence of osteoarthritis of the hip joint in dogs. Journal of the American Veterinary Medical Association 229: 690-693.

Thrusfield M (1995) The experimental population. In: Veterinary Epidemiology, pp 260.

Welsh EM, Gettinby G, Nolan AM (1993) Comparison of a visual analogue scale and a numerical rating scale for assessment of lameness, using sheep as a model. American Journal of Veterinary Research 54: 976-983. 